\title{
Bifurcations of the spatial central configurations in the 5-body problem
}

\author{
Martha Alvarez-Ramírez, Motserrat Corbera, and Jaume Llibre \\ Departamento de Matemáticas \\ UAM-Iztapalapa. \\ 09430 Iztapalapa \\ México, D.F. México \\ mar@xanum. uam.mx \\ Departament de Tecnologies Digitals i de la Informació \\ Universitat de Vic. \\ C/. Laura, 1308500 Vic, Barcelona \\ Catalonia, Spain \\ montserrat. corbera@uvic.cat \\ Departament de Matemàtiques \\ Edifici C, Facultat de Ciències \\ Universitat Autònoma de Barcelona \\ 08193 Bellaterra (Barcelona), Spain \\ jllibre@mat.uab.cat
}

\section{Introduction}

A configuration of $n$ particles is called central when the acceleration vector of each particle is a common scalar multiple of its position vector. One of the reasons why central configurations are interesting is that they allow us to obtain explicit homographic solutions of the $n$-body problem, that is, motions where the configuration of the system changes size but keeps its shape. Also, they are important in the study of total collisions.

Even the finiteness of the number of central configurations is a very difficult question. This conjecture was proposed by Chazy $[\mathbf{7}]$ and Wintner $[\mathbf{2 1}]$ and was listed by Smale as problem number 6 on his list of problems for this century [18]. Central configurations, which appear so deeply in the dynamics of the $n$ body problem are very difficult to count [18]. A complete enumeration of all such solutions for $n \geq 4$ represents a very difficult task for the present day methods.

For the collinear $n$-body problem, an exact count of the central configurations of $n$ bodies was found by Moulton (1910). He showed that there are $n ! / 2$ equivalence classes.

Saari [19] proved that the regular $N-1$ dimensional simplex is a central configuration of $N$ bodies for any value of the masses. In particular, case $N=4$ has been well known over a century (Lehmann-Filhés 1891,[11]). The fact that the tetrahedron is the unique spatial central configuration of four bodies was proved by Pizzetti (1904, [16]).

The number of planar central configurations of the $n$-body problem for an arbitrary given set of positive masses has been established only for $n=3$, namely, Euler's three collinear configurations and Lagrange's two equilateral triangle configurations. For $n=4$, Hampton and Moeckel [9] showed that, in addition to the tetrahedral spatial configurations, there are only finitely many equivalence classes of planar central configurations.

For $n=5$, Hampton and Jensen [10] with computer assistance showed the finiteness of the spatial central configurations with positive masses, with the exception of some 
explicit special cases of mass values, and Albouy and Kaloshin [3] have proved that the planar 5-body central configurations are finite apart from some explicitly given special cases. For $n \neq 6$, it is not known, in general, if the number of equivalence classes of central configurations is finite. The finiteness question and a number of other interesting questions on central configurations are discussed in a recent problem list given in Albouy et al. $[\mathbf{2}]$.

In this paper we analyze the families of central configurations of the spatial 5 -body problem with four masses equal to 1 and the fifth mass, $m$, varying from 1 to 0 . In particular we will find two bifurcation values of $m$. This is accomplished by using bifurcation theory and the method of analytical continuation to follow numerically the central configurations as the mass parameter $m$ varies, and we use symbolic computation software Mathematica ${ }^{\circledR}$ to handle the more tedious calculations.

\section{Spatial central configurations in the 5-body problem}

In this section we will describe the setting of the newtonian 5-body problem in Euclidean three-space and we give the equations of central configurations of 5 bodies in $\mathbb{R}^{3}$.

We start by considering the spatial 5 -body problem

$$
m_{i} \ddot{\mathbf{q}}_{i}=-\sum_{\substack{j=1 \\ j \neq i}}^{5} G m_{i} m_{j} \frac{\mathbf{q}_{i}-\mathbf{q}_{j}}{\left|\mathbf{q}_{i}-\mathbf{q}_{j}\right|^{3}},
$$

$i=1, \ldots, 5$, where $\mathbf{q}_{i} \in \mathbb{R}^{3}$ is the position vector of the punctual mass $m_{i}$ in an inertial coordinate system and $G$ is the gravitational constant which can be taken equal to one. The configuration space of the spatial 5-body problem is defined as

$$
\mathcal{P}=\left\{\left(\mathbf{q}_{1}, \ldots, \mathbf{q}_{5}\right) \in \mathbb{R}^{15}: \mathbf{q}_{i} \neq \mathbf{q}_{j}, \text { for } i \neq j\right\} .
$$

Given masses $m_{1}, \ldots, m_{5}$, the corresponding configuration $\left(\mathbf{q}_{1}, \ldots, \mathbf{q}_{5}\right) \in \mathcal{P}$ is central if the acceleration vector for each body is a common scalar multiple of its position vector (with respect to the center of mass). That is, if there exists a positive constant $\lambda$ such that

$$
\ddot{\mathbf{q}}_{i}=-\lambda\left(\mathbf{q}_{i}-\mathbf{q}_{m}\right),
$$

for $i=1, \ldots, 5$, where $\mathbf{q}_{m}$ is the position vector of the center of mass of the system given by

$$
\mathbf{q}_{m}=\frac{\sum_{i=1}^{5} m_{i} \mathbf{q}_{i}}{\sum_{i=1}^{5} m_{i}}
$$

Therefore, the configuration $\left(\mathbf{q}_{1}, \ldots, \mathbf{q}_{5}\right) \in \mathcal{P}$ of the 5 -body problem with positive masses $m_{1}, \ldots, m_{5}$ is central if there exists $\lambda$ such that $\left(\lambda, \mathbf{q}_{1}, \ldots, \mathbf{q}_{5}\right)$ is a solution of the system

$$
\lambda\left(\mathbf{q}_{i}-\mathbf{q}_{m}\right)=\sum_{\substack{j=1 \\ j \neq i}}^{5} m_{j} \frac{\mathbf{q}_{i}-\mathbf{q}_{j}}{\left|\mathbf{q}_{i}-\mathbf{q}_{j}\right|^{3}}, \quad \text { for } \quad i=1, \ldots, 5 .
$$

We choose the coordinates for the body with mass $m_{i}$ as $\mathbf{q}_{i}=\left(x_{i}, y_{i}, z_{i}\right)$ for $i=$ $1, \ldots, 5$. Without loss of generality we assume that the body with mass $m_{1}$ is fixed at $\left(x_{1}, y_{1}, z_{1}\right)=(0,0,1)$ and that $x_{3}=0$, the last assumption is to avoid the rotation with 
Figure 1. Equal mass spatial 5-body central configurations. These figures were taken from $[\mathbf{1 4}]$.

respect to the $z$-axis. By taking the center of mass at $(0,0,0)$, system $(3)$ can be written as

$$
\mathbf{f}_{i}=\sum_{\substack{j=1 \\ j \neq i}}^{5} m_{j} \frac{\mathbf{q}_{i}-\mathbf{q}_{j}}{\left|\mathbf{q}_{i}-\mathbf{q}_{j}\right|^{3}}-\lambda \mathbf{q}_{i}=0, \quad \text { for } \quad i=1, \ldots, 5 .
$$

It is easy to check that

$$
m_{1} \mathbf{f}_{1}+m_{2} \mathbf{f}_{2}+m_{3} \mathbf{f}_{3}+m_{4} \mathbf{f}_{4}+m_{5} \mathbf{f}_{5}=0 .
$$

Assume now that $m_{2} \neq 0$, from $(5)$ the vectorial equation $\mathbf{f}_{2}$ is a linear combination of the other ones and it can be eliminated. Moeover since the center of mass is fixed at the origin, from (2) we get

$x_{2}=-\frac{m_{4} x_{4}+m_{5} x_{5}}{m_{2}}, y_{2}=-\frac{m_{3} y_{3}+m_{4} y_{4}+m_{5} y_{5}}{m_{2}}, z_{2}=-\frac{m_{1}+m_{3} z_{3}+m_{4} z_{4}+m_{5} z_{5}}{m_{2}}$.

Finally we isolate $\lambda$ from the first component of the vectorial equation $\mathbf{f}_{1}$ and we substitute it into the other equations. In short system (4) has been simplified to a set of eleven equations which depend on eight unknowns, namely, the position variables $y_{3}, z_{3}, x_{4}$, $y_{4}, z_{4}, x_{5}, y_{5}, z_{5}$. Clearly the eleven equations obtained are not all independent. Then in order to have a central configuration, we are seeking the zeros of the system of eight equations in the eight unknowns that satisfy the remaining three equations. Since, equations obtained are essentially nonlinear, their solutions must be done combining numeric and symbolic computations, which are carried out using Mathematica ${ }^{\circledR}$, is just a good tool for doing such calculations.

\section{Some previously known results}

The main impulse for our study comes from previous results which are described below.

\subsection{Central configuration for the 5-body problem with equals masses}

Kotsireas and Lazard [12] assumed that non-planar equal mass central configurations of 5 bodies always have several symmetries and used linear algebra and Gröbner bases to classify symmetric spatial central configurations of 5 bodies with equal masses. They conjectured that there are only four three-dimensional central configurations of 5 bodies with equal masses (up to isometry, rescaling and permutation of the particles), namely two convex and two concave (see Figure 1). Alvarez-Ramírez et al. [6] and Santoprete and Lee $[\mathbf{1 4}]$ succeeded in proving the conjecture to be correct.

\subsection{Central configuration in the $(4+1)$-body problem}

The $(4+1)$-body problem is a particular case of the 5 -body problem with 4 equal masses and an infinitesimal mass. Next we summarize the known results of this problem. In 
[17] the author shows that a configuration with four equal masses located at the vertices of an equilateral tetrahedron and an infinitesimal mass at its barycenter is a central configuration. Almeida [5] proved that for the $(4+1)$-body problem there are 25 central configurations with the four positive equal masses forming a tetrahedron, among which 12 are non-convex. They provide 6 different classes of central configurations up to isometry, rescaling and permutation of the particles. Later, Tsai $[\mathbf{2 0}]$ found the same result using Gröebner bases.

It is well known that four equal masses in the plane are necessarily in non-collinear central configuration, namely, a square, an equilateral triangle with a mass at its center, and a particular isosceles triangle with another mass on its axis of symmetry, see [1]. We see that each one of these central configurations provides a central configuration of the spatial $(4+1)$-body problem with four equal finite masses contained in the same plane and the fifth particle with infinitesimal mass lying out of this plane.

In short we have 9 different classes of central configurations of the $(4+1)$-body problem.

\section{Bifurcation analysis}

We continue numerically via the analytic continuation method the central configurations of the 5-body problem with equal masses to the $(4+1)$-body problem and viceversa. We note that the four classes of central configurations of the 5-body problem with 5 equal masses can be continued to 9 different classes of the 5-body problem with four equal masses and the fifth mass close to 1, depending on the position of the mass that is continued to values different from 1 . We find two critical values, one is the well known bifurcation mass value $m_{c}=\frac{10368+1701 \sqrt{6}}{54952} \approx 0.264496 \ldots$ while the second one is given by $m_{f} \approx 0.66 \ldots$

Our main result is summarized as follows.

Theorem 1 There are 5 different families of central configurations connecting the 5-body problem with equal masses and the $(4+1)$-body problem without bifurcation.

There are 2 families of central configurations starting at the 5-body problem with equal masses and ending at the bifurcation point with $m=m_{c}$ and 2 additional families ending at the bifurcation point $m_{f}$.

There are 3 families of central configurations starting at the $(4+1)$-body problem and ending at the bifurcation point with $m=m_{c}$ and 1 additional family ending at the bifurcation point $m_{f}$.

\section{References}

[1] Albouy, A. Symétrie des configurations centrales de quatre corps., C. R. Acad. Sci. Paris Sér. I Math. 320 (1995), no. 2, 217-220.

[2] Albouy, A., Cabral, H., Santos, A.: Some problems on the classical $n$-body problem. Celest. Mech. Dyn. Astron. 113 (2012), 369-375.

[3] Albouy, A. and Kaloshin, V. Finiteness of central configurations of five bodies in the plane, Ann. of Math. (2) 176 (2012), no. 1, 535-588.

[4] Albouy A.and J. Llibre, J. Spatial central configurations for the $1+4$ body problem, Contemp. Math., 292 (2002), 1-16.

[5] Almeida Santos, A. Dziobek's configurations in restricted problems and bifurcation. Celestial Mech. Dynam. Astronom. 90 (2004), no. 3-4, 213-238. 
[6] Alvarez, M., Delgado, J., Llibre, J. On the spatial central configurations of the 5-body problem and their bifurcations. Discrete Contin. Dyn. Syst. Ser. S 1 (2008), no. 4, 505-518.

[7] Chazy, J. Sur certaines trajectoires du probleme des $n$ corps, Bull. Astron. 35 (1916, 1918), 321-389.

[8] Hampton, M. Generic finiteness for Dziobek configurations, Trans. Amer. Math. Soc. 353 (2001), 4673-4686.

[9] Hampton, M., Moeckel, R. Finiteness of relative equilibria of the four-body problem. Invent. Math. 163 (2006), 289-312.

[10] Hampton, M. and Jensen, A. Finiteness of spatial central configurations in the five-body problem. Celestial Mech. Dynam. Astronom. 109 (2011), no. 4, 321-332.

[11] Lehmann-Filhés, R. Ueber zwei Fälle des Vielkörperproblems. Astron. Nachr. 127, 137-143 (1891).

[12] Kotsireas I. and Lazard, D. Central configurations of the 5-body problem with equal masses in three-dimensional space. Zap. Nauchn. Sem. S.-Peterburg. Otdel. Mat. Inst. Steklov. (POMI) 258 (1999), Teor. Predst. Din. Sist. Komb. i Algoritm. Metody. 4, 292-317, 360-361; translation in J. Math. Sci. (New York) 108 (2002), no. 6, 11191138.

[13] Leandro, E. S.G. On the Dziobek configurations of the restricted $(N+1)$-body problem with equal masses, Discrete Contin. Dyn. Syst. Ser. S 1 (2008), no. 4, 589-595.

[14] Lee, T. L. and Santoprete, M. Central configurations of the five-body problem with equal masses. Celestial Mech. Dynam. Astronom. 104 (2009), no. 4, 369-381.

[15] Moeckel, R. Finiteness of spatial central configurations in the five-body problem, Celestial Mech. Dynam. Astronom. 109 (2011), no. 4, 321-332.

[16] Pizzetti, P. Casi particolari del problema dei tre corpi. Rendiconti 13 (1904), 17-26.

[17] Schmidt, D. S. Central configurations in $\mathbb{R}^{2}$ and $\mathbb{R}^{3}$. Hamiltonian dynamical systems (Boulder, CO, 1987), 59-76, Contemp. Math., 81, Amer. Math. Soc., Providence, RI, 1988.

[18] Smale, S. Mathematical problems for the next century, Math. Intell. 20 (1998), 7-15.

[19] Saari, D. On the role and properties of $n$-body central configurations. Celest. Mech. 21, 9-20 (1980)

[20] Tsai, Y. Dziobek configurations of the restricted $(N+1)$-body problem with equal masses, J. Math. Phys. 53 (2012), no. 7, 072902.

[21] Wintner, A. The Analytical Foundations of Celestial Mechanics. Princeton Mathematical Series, v. 5, Princeton Univ. Press, Princeton, NJ, 1941. 